\title{
Upregulated FGFRI expression is associated with the transition of hormone-naive to castrate-resistant prostate cancer
}

\author{
K Armstrong', I Ahmad ${ }^{2,3}$, G Kalna ${ }^{2}$, SS Tan ${ }^{2,3}$, J Edwards ${ }^{3}$, CN Robson' and HY Leung ${ }^{*, 1,2,3}$ \\ 'Solid Tumour Target Discovery Group, Northern Institute for Cancer Research, Newcastle University, Newcastle NE2 4HH, UK; ${ }^{2}$ Urology Research, \\ Beatson Institute for Cancer Research, Garscube Estate, Switchback Road, Glasgow G6I IBD, UK; ${ }^{3}$ Experimental Therapeutics, Institute for Cancer \\ Studies, University of Glasgow, Glasgow GI 2 8QQ UK
}

BACKGROUND: Prostate cancer (PC) represents a global health issue. Treatment for locally advanced and metastatic PC remains unsatisfactory. The androgen receptor (AR) has been validated in having a key role in both naïve and castrate-resistant PC (CRPC) However, the significance of other signalling pathways in CRPC is less well validated.

METHODS: To gain a better insight into the molecular signalling cascades involved in clinical CRPC, we performed gene expression profiling using the Illumina DASL assay and studied matched hormone-naive (HN) and CR prostate tumours ( $n=10$ pairs). Ingenuity Pathways Analysis (IPA) was used to identify potential networks involved, and further validation was performed in in vitro cell models and clinical tumours.

RESULTS: Expression of 50 genes was significantly different between HN and CRPC. IPA revealed two networks of particular interest,

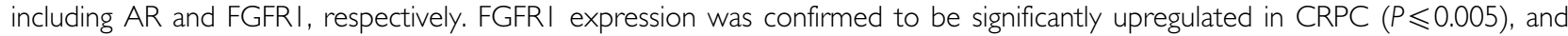
abnormal FGFRI expression was associated with shorter time to biochemical relapse in HNPC $(P=0.006)$ and less favourable disease-specific survival in CRPC $(P=0.018)$.

CONCLUSION: For the first time, our gene expression profiling experiment on archival tumour materials has identified upregulated FGFRI expression to be associated with PC progression to the CR state.

British Journal of Cancer (201 I) I 05, I362-1369. doi:10.1038/bjc.201 I.367 www.bjcancer.com

Published online 27 September 2011

(c) 201 I Cancer Research UK

Keywords: prostate cancer; formalin-fixed, paraffin-embedded samples; gene expression analysis; hormone-naive and castrate-resistant prostate cancer; fibroblast growth factor receptor I

Prostate cancer (PC) is the most commonly diagnosed cancer in men in the developed world, and its incidence continues to rise in most countries (Ferlay et al, 2007). There is a major unmet need to improve treatment for patients who develop castrate-resistant PC (CRPC), which remains incurable (Scher et al, 2005). Recent studies have confirmed involvement of persistent androgen receptor (AR) signalling in CRPC (Watson et al, 2010). The significance of this is further supported by the demonstrated clinical benefit with androgen ablation achieved by abiraterone treatment (Reid et al, 2010). However, how signalling cascades interact and modulate $\mathrm{AR}$ to contribute to CRPC remains poorly understood. In addition, non-AR-mediated signalling in CRPC has been predominantly studied in pre-clinical models, using in vitro cell studies and in vivo animal models. These studies have proposed the involvement of multiple signalling cascades and tumour suppressor genes in the pathogenesis of CRPC (Aggarwal and Ryan, 2011). However, the clinical relevance of many of these pathways remains to be validated. Archival formalin-fixed, paraffin-embedded (FFPE) tissue and associated clinical data provide a valuable resource to link gene expression data with tumour biology and patient outcome. We applied the cDNA-mediated annealing, selection, extension and ligation

*Correspondence: Professor HY Leung; E-mail: h.leung@beatson.gla.ac.uk Received 18 April 2011; revised II August 2011; accepted 17 August 20I I; published online 27 September 20I I
(DASL) assay (Illumina, San Diego, CA, USA; Bibikova et al, 2008) in paired hormone-naive (HN) and CR prostate tumour samples. Our objective was to identify key pathways implicated in the evolution of CR disease.

\section{MATERIALS AND METHODS}

\section{Cell lines and reagents}

LNCaP cells were obtained from the American Type Culture Collection (Manassas, VA, USA) and maintained in RPMI 1640 media supplemented with $10 \%$ fetal calf serum (Sigma, St Louis, MO, USA) at $37^{\circ} \mathrm{C}$ in $5 \% \mathrm{CO}_{2}$ atmosphere. CdxR-LNCaP cells were generated and maintained as described previously (Halkidou et al, 2003; Rigas et al, 2007). Anti-FGFR1 antibody (Flg (C-15)) was obtained from Santa Cruz Biotechnologies (Santa Cruz, CA, USA).

\section{RNA isolation and clinical samples}

RNA was isolated from cell lines using TRIzol reagent (Invitrogen, Carlsbad, CA, USA) then treated with DNAfree (Ambion, Austin, TX, USA) to remove any contaminating DNA according to the manufacturer's protocol.

Gene expression analysis was performed on matched $\mathrm{HN}$ and LHRH-relapsed (or CR) samples obtained by transurethral 
resection of the prostate (TURP) from 10 patients. Biochemical relapse signifying CRPC was defined both biochemically and histologically. The cohort of patients for $\mathrm{HN}$ and CR cancer were selected for analysis if they initially responded to hormone treatment (response was defined by prostate-specific antigen (PSA) levels decreasing by at least $50 \%$ ), but subsequently relapsed (two consecutive increases in PSA of $>10 \%$ ) and had a prehormone and post-hormone relapse sample available for analysis. All patients received hormone therapy in the form of androgen deprivation therapy (either subcapsular bilateral orchidectomy and GnRH analogue) or maximum androgen blockade. To meet the inclusion criteria for matched-HN and -CR tumours, a response to this therapy had to be observed; a response to this therapy was defined by the PSA levels decreasing by at least $50 \%$, and a nadir being reached. The hormone-sensitive tumour samples were obtained either from a TURP or prostatic needle biopsies. In addition, patients were required to relapse with CRPC to meet the inclusion criteria. A tumour was classified as CR if the patient stopped responding to hormone therapy, which was signified by two consecutive increases in their PSA levels. CR tumour samples were obtained by TURP, and all patients in this cohort initially respond to hormone therapy, but subsequently relapsed with hormonerefractory disease. All clinical samples were used in accordance with approval granted by the local hospital ethics committee.

FFPE tissue specimens were analysed histologically to identify tumour-rich regions, and samples $(n=3)$ were taken using a $1-\mathrm{mm}$ diameter core punch with the MTA-01 personal tissue arrayer (Beecher Instruments, Sun Prairie, WI, USA). Cores were deparaffinised in xylene $(2 \times 10 \mathrm{~min})$, re-hydrated in $100,90,70$ and $50 \%$ ethanol ( 5 min each) then air-dried. Tissues were digested in $100 \mu \mathrm{l}$ Proteinase $\mathrm{K}$ digestion buffer with $15 \mu$ l Proteinase K (60 $\mathrm{U} \mu \mathrm{l}^{-1}$ ) (Ambion) at $55^{\circ} \mathrm{C}$ overnight to remove cross-links. RNA was isolated using the HighPure RNA paraffin kit (Roche, Branchburg, NJ, USA), which includes a DNase I step to remove contaminating DNA.

\section{DASL assay and the IPA}

Generation of cDNA, DASL assay, hybridisation to the bead array and data collection were carried out as described previously (Fan et al, 2004; Bibikova et al, 2004a,b). Results were analysed using the BeadStudio software (Illumina), with data normalised using the Cubic Spline method as described by Bibikova et al, (2004a, b).

All detected genes were individually analysed. Genes with detection scores $>0.99$ were included in the analysis (detection score $=1-P$-value, determined whether the target sequence signal was distinguishable from the negative control). Genes were then determined as significantly altered if their DiffScore exceeded 13, which signifies $P<0.05$. The DiffScore statistic assesses the probability of differential expression between a reference sample and a condition on an array. Within this model, technical and biological errors are taken into account. Technical error was determined using a least squares fit of s.e. and mean for the beads on both the reference and condition arrays. Average intensity of negative controls on reference and condition arrays provided an estimate of biological variation. Furthermore, a $P$-value was determined by dividing the absolute difference between the expression reference and condition arrays by the sum of the technical and biological variation on both arrays. Therefore, DiffScore $=10 \times \operatorname{Sign}\left(\operatorname{cond}_{\text {mean }}-\mathrm{ref}_{\text {mean }}\right)$ log_10 (p) (Chudin et al, 2006).

The 'Core Analysis' from IPA (Ingenuity System Inc., Redwood City, CA, USA; http://www.ingenuity.com/) was used to interpret the set of 50 genes with DiffScore $>13(P<0.05)$, either up- or downregulated between $\mathrm{HN}$ and CRPC. IPA identified a set of networks involving subgroups of genes from the 50 identified genes.

\section{Primer design and qRT-PCR}

Reverse transcription was performed on 2- $\mu \mathrm{g}$ RNA extracted from cell lines. Briefly, $0.4 \mathrm{mM}$ dNTP mix (Sigma), $0.25 \mu \mathrm{g}$ Oligo d(T) ${ }_{15}$ (R\&D Systems, Minneapolis, MN, USA) and 100 units M-MLV reverse transcriptase (Promega, Madison, WI, USA) was used in a 20- $\mu \mathrm{l}$ reaction. Reactions were performed at $37^{\circ} \mathrm{C}$ for $1 \mathrm{~h}$ followed by enzyme inactivation at $95^{\circ} \mathrm{C}$ for $5 \mathrm{~min}$, immediately followed by incubation on ice.

Primers were designed using Primer Express (Applied Biosystems, Carlsbad, CA, USA), restricting amplicons to intron/ exon boundaries and products approximately $50 \mathrm{bp}$ in length. Reaction efficiencies were between $90-110 \%\left(R^{2}>0.98\right)$ and all primers displayed one peak upon melting curve analysis. Primers against FGFR1 were designed to detect all the nine alternative transcripts. All primers used were provided by VHBio (Gateshead, UK). qRT-PCR was carried out using SYBR Green Jumpstart Taq Readymix (Sigma) in 384-well clear optical reaction plates, using the ABI 7900HT real-time PCR system (Applied Biosystems) according to the manufacturer's protocol. Primers were used at a final concentration of $1 \mathrm{ng} \mu \mathrm{l}^{-1}$. All cell line samples were tested in triplicate at least twice. Expression was normalised against HPRT1 expression and repeated twice. Data generated was analysed by absolute quantification using SDS v2.2 software (Applied Biosystems). Primer sequences are as follows: RPL13a (5'-GTA CGC TGT GAA GGC ATC AA-3; 5'-GTT GGT GTT CAT CCG CTT G-3), FGFR1 (5'-ACA CCA AAC CAA ACC GTA TG-3'; $5^{\prime}$-TGT CCA ATA TGG AGC TAC GGG-3'), and HPRT1 (5'-TTG CTT TCC TTG GTC AGG CA- $3^{\prime}$; $5^{\prime}$-AGC TTG CGA CCT TGA CCA TCT $\left.-3^{\prime}\right)$.

\section{Immunohistochemistry (IHC)}

IHC was performed on FFPE sections using Flg (C-15) (Santa Cruz Biotechnologies), an antibody which has been fully validated (Berger et al, 1999). A PC tissue microarray (TMA, ethical committee approval, MREC 01/0/36) was used, comprising 223 patients: 164 primary PC, 23 benign prostatic hypertrophic (BPH) and 36 matched pairs of $\mathrm{HN}$ and CR tumour samples.

For the HN- and CR-matched TMA, the median age of the patients at diagnosis was 70 , ranging from 49 to 81 , and the median PSA at diagnosis was $32 \mathrm{ng} \mathrm{ml}^{-1}$ (range $3-126$ ) with a median Gleason sum score for the HN tumours at 7 (ranging from 4 to 10). At diagnosis, 12 patients had metastatic disease; however, this increased to 28 at relapse. The median time to relapse was 2.66 years (inter quartile range 1.76-4.71). Follow-up data were available for 33 patients. The median time to death from relapse was 1.87 years (interquartile range $1.05-2.93$ ), whereas the median time to death from diagnosis was 5.82 years (interquartile range 3.44-6.83). During the follow-up period, by definition, all patients in this cohort relapsed with CR disease; 25 died of their disease and 8 deaths were attributed to intercurrent disease. An incidence TMA of PC was used to compare FGFR1 expression between PC and $\mathrm{BPH}$ controls. The median age of patients at diagnosis was 71.2 years (minimum of 48.3 years, maximum of 92.4 years). Median Gleason score of the prostate tumours was 7 (minimum of 2, maximum of 9).

Expression of FGFR1 in each core was assessed using the weighted histoscore method (Edwards et al, 2003), grading staining intensity as negative (0), weak (1), moderate (2) and strong (3), before multiplying by the percentage of tumour cells within each category. The final histoscores ranged from a minimum of 0 to a maximum of 300 . Quantification of high/low was defined as above or below the median, respectively. This was graded separately by two observers (SST and IA), blinded to all outcome data. Inter-observer agreement was excellent with interclass correlation scores $>0.80$. 


\section{Statistical analysis}

Statistical evaluation was performed using the Mann - Whitney test to compare differences in FGFR1 expression between cancer and benign control samples. Paired-sample analysis was used to compare changes in protein expression between $\mathrm{HN}$ and $\mathrm{CR}$ samples. As individual pairs of these samples were from the same patient and were therefore related, it was considered that the Wilcoxon signed-rank test was an appropriate analysis to be used. Disease-specific survival rates were generated using the KaplanMeier method. The log-rank test was used to compare significant differences between the staining intensities. The Pearson correlation coefficient was used to test for correlation. Analyses were performed using the Statistical Package for Social Sciences software (Version 17.0; SPSS, Chicago, IL, USA).

\section{RESULTS}

Gene expression analysis to compare clinical HN- and CRPC

Ten matching pairs of $\mathrm{HN}$ and CRPC samples were studied. RNA isolated using the HighPure RNA Paraffin Kit (Roche) was quantified using RiboGreen (Invitrogen) with concentrations ranging between $66-324 \mathrm{ng} \mu \mathrm{l}^{-1}$, satisfying the required minimal concentration $\left(50 \mathrm{ng} \mu \mathrm{l}^{-1}\right)$ for DASL assay. In addition to RNA assay by the Agilent Bioanalyser (Agilent Technologies, Santa Clara, CA, USA), qRT-PCR was performed on all samples for ribosomal protein L13a (RPL13a) to generate a 90-bp fragment. The observed $\mathrm{Ct}$ values of 22 to 28 cycles excluded excessive degradation (Figure 1A) as determined by Bibikova et al, (2008). All samples were comparable in terms of their degradation status and no alterations in array quality were observed between samples. Analysis of matched-HN and -CRPC samples were performed over two arrays (Human Cancer Panel v1 containing 502 genes; Illumina), where matched pairs were applied to the same array, along with the relevant technical replicates and hybridisation controls. A linear relationship $\left(R^{2}=0.998\right.$; Figure $\left.1 \mathrm{~B}\right)$ was observed between the two data sets, signifying data comparability. Overall, 456 genes, out of 502 cancer-related genes within the cancer panel, were detected on both arrays with a detection score $>0.99$ (as defined in Materials and Methods). Cluster analysis of the data without normalisation confirmed that all samples were highly correlated with $R>0.945$ (Figure 1C).

Among the 456-detected genes, the expression of 50 genes were significantly different between $\mathrm{HN}$ and CR tumours (DiffScore $>13 ; P<0.05)$ as determined using BeadStudio software, with 21 genes upregulated and 29 genes downregulated (Figure 2). Thirteen genes were upregulated in CR tumours by more than twofold, including $A R, C A V 1, E T S 2, F G F R 1, I G F 2, I G F-B P 3, M M P 7$, TIMP3 and TNFRSF5, whereas 16 genes were downregulated by more than two-fold, including $A K T 1, C D K 7, E R B B 3, I G F 1, I G F-$ $B P 5$, and $V E G F$. Interestingly, molecules within the same signalling pathway can be differentially regulated during the $\mathrm{HN}$ to $\mathrm{CR}$ transition, including FGFR1 $(\uparrow)$ and FGFR3 $(\downarrow)$, IGF2/IGF-BP3 ( $\uparrow)$ and IGF1/IGF-BP5 $(\downarrow)$, highlighting potential complex relationship between members of individual signalling pathways in CR prostate carcinogenesis.

To carry out formal assessment of the global signalling abnormalities associated with the development of CR disease, the 'core analysis' from IPA software (http://www.ingenuity.com/) was performed on the 50 differentially expressed genes between HN and CR tumours. IPA identified six heuristic networks that contain $13,12,12,7,5$ and 2 genes from the list of 50 -identified genes, respectively. The top ranked network involved 13 of the 50 genes (network 1; Figure 3A; Supplementary Table) including AR; the top functions associated with this network are tissue development, cell cycle and gene expression. The second ranked network implicated
A

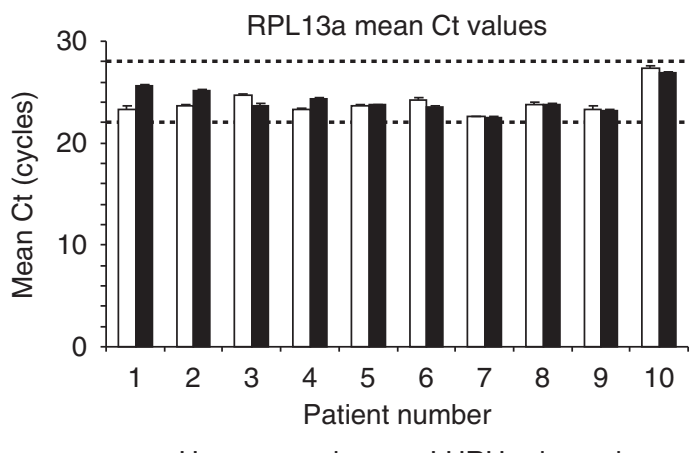

B

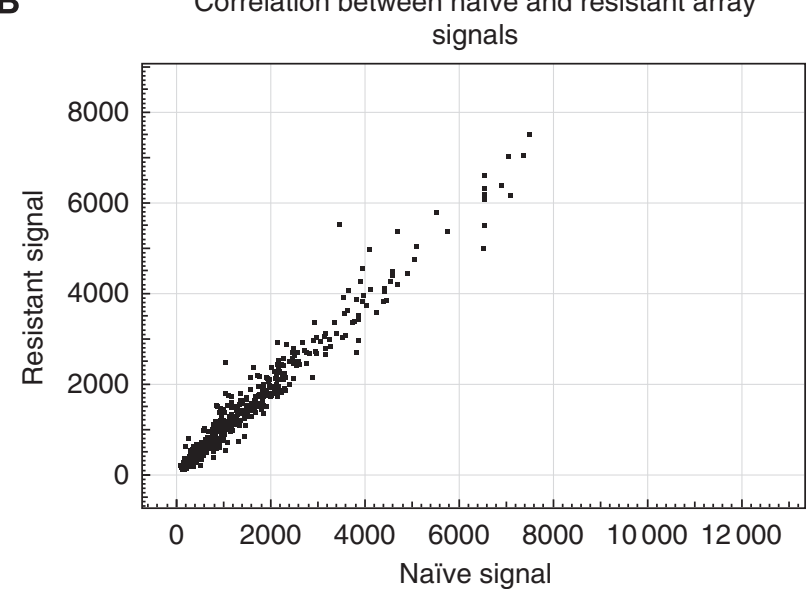

C Cluster analysis of unnormalised data

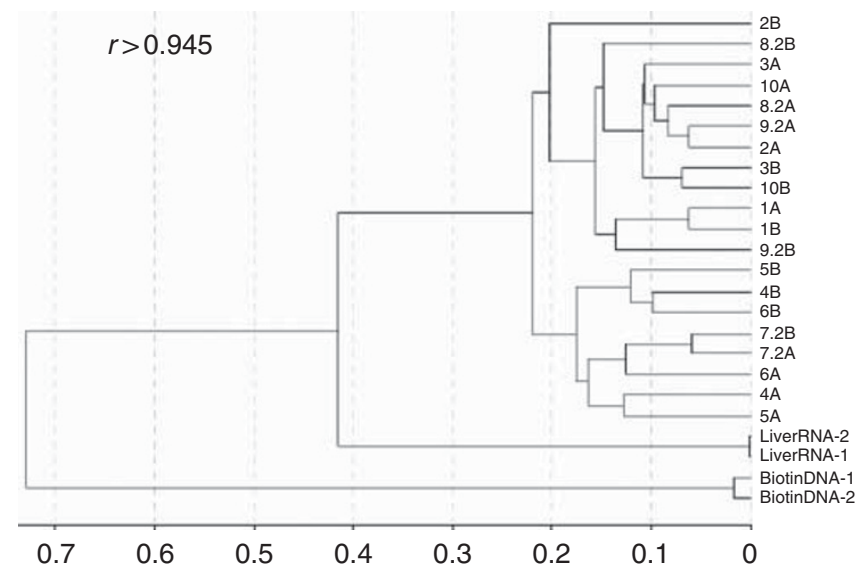

Figure I Pre-qualification of samples and evaluation of technical array data. (A) RPLI3a expression in 10 paired hormone-naive and $\mathrm{LHRH}$ relapsed samples. RPL I 3a expression was determined by qRT-PCR. Data is expressed as mean Ct values \pm s.d. qRT-PCR was performed in triplicate $(n=2)$. (B) Naïve and resistant sample groups show a linear relationship. (C) Cluster analysis of unnormalised data reveals that all samples are highly correlated.

in the $\mathrm{HN}$ to CR transition identified 12 of the 50 genes, six of which were upregulated (Figure 3B; network 2 in Supplementary Table); the top functions of network 2 are tumour morphology, developmental disorder and genetic disorder. A third network, referred to as network 3, which is highly enriched for nuclear receptor network components, and identified 12 genes, but only three of these genes were upregulated, namely IGF-BP3, IGF2 and Runx1T (Figure 3C; Supplementary Table). We are particularly 


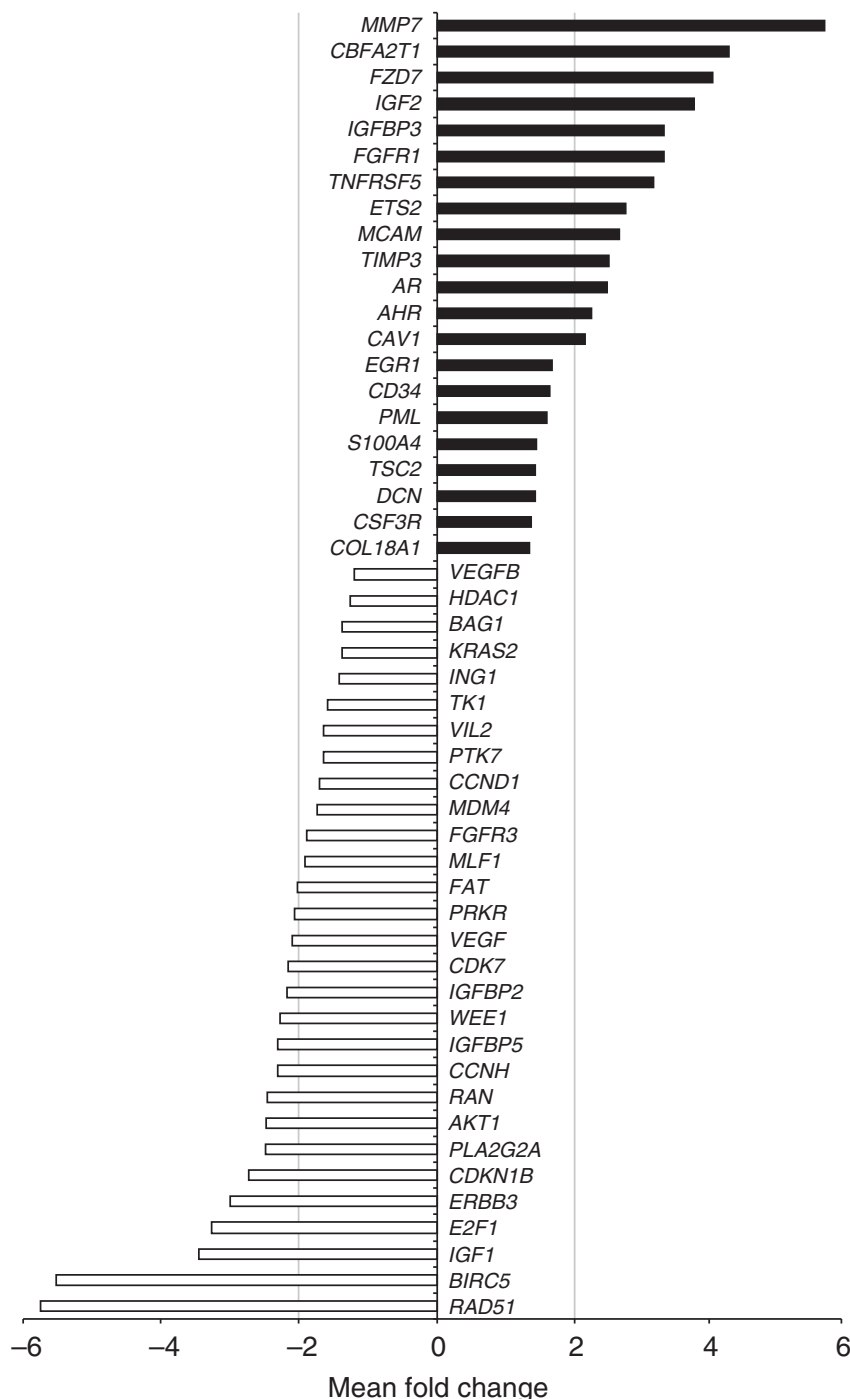

Figure 2 Differentially expressed genes between hormone-naive (HN) and castrate-resistant (CR) tumours. Fifty genes were determined as differentially expressed (DiffScore > 13). Solid and open bars represent genes upregulated or downregulated, respectively, during transition from $\mathrm{HN}$ to $\mathrm{CR}$ status. The mean fold change in gene expression is represented in the $x$ axis.

interested to identify novel oncogenic activation associated with the transition from HN to CRPC. Hence, networks 1 and 2 are particularly interesting to our project.

Within network 1, AR appears to be critically involved as a signalling hub interacting with the majority of implicated genes in this network. In addition, $A R$ has been extensively investigated and validated in both HN and CR disease (Shen and Balk, 2009). Hence, our focus turned to network 2. Twelve genes were implicated in network 2; the upregulated genes were EGR1, FGFR1, MCAM, TSC2, S100 and S100A4, whereas FGFR3, VEGFa/b, ras, MDM4 and TK1 were downregulated. Within the FGFR system both FGFR1 and FGFR3 are highlighted in this network. Significantly, the degree of upregulation for FGFR1 at $\sim$ three-fold was highest within network 2. As expected, FGFR1 signalling closely links to ERK1/2. FGFR1 can also crosstalk with other receptor tyrosine kinases, including FGFR3 and erbB3. It is worth noting that the expression of $V E G F a / b$ was reduced in CRPC. FGF- and VEGFmediated signalling critically controls angiogenesis during tumourigenesis. VEGFR and FGFR may co-operate to promote neo-angiogenesis. Therefore, upregulated FGFR1 expression may compensate for the reduced expression of $V E G F a / b$ observed in network 2. Work from our group and others have previously implicated abnormal FGFR1 expression in prostate carcinogenesis (Devilard et al, 2006; Acevedo et al, 2007; Sahadevan et al, 2007). However, the role of FGFR1 in the development of CRPC has not been previously described. Overall, network 2 appeared to be novel, and we wish to validate the potential role of FGFR1 in both $\mathrm{HN}$ and CRPC, as its upregulation is ranked highest in this network. Our data therefore suggest that FGFR1 may also have a role in CRPC. We further evaluated whether FGFR1 expression is upregulated during the transition from $\mathrm{HN}$ to $\mathrm{CR}$ state using an in vitro cell model and clinical CRPC materials.

\section{FGFR1 in in vitro and clinical CRPC}

Parental LNCaP cells were cultured continuously in the presence of $2 \mu \mathrm{M}$ bicalutamide (or casodex) for over 9 months. This resulted in the bicalutamide- (or casodex)-resistant phenotype; the bicalutamide-resistant LNCaP (referred to as cdxR-LNCaP thereafter) cells can be considered a model of CRPC (Rigas et al, 2007). In these cdxR-LNCaP cells with chronic AR antagonist treatment, FGFR1 expression was upregulated by about 2.5-fold (Figure 4A). Similarly, FGFR1 mRNA expression was upregulated by two-fold when AR function in LNCaP cells was acutely blocked by bicalutamide $(2 \mu \mathrm{M})$ for $48 \mathrm{~h}$. These in vitro data is in keeping with the notion that abnormal FGFR1 expression is associated with CRPC, as well as HN disease.

Using IHC, prostate TMA containing $164 \mathrm{PC}$ and $23 \mathrm{BPH}$ samples were studied for FGFR1 expression (Figure 4B). We were able to validate our previous report of upregulated FGFR1 expression in $\mathrm{PC}$, when compared with benign (BPH) control samples $(P<0.0001$; Sahadevan et al, 2007). To test the role of FGFR1 in CRPC, a TMA containing matched HN and CR tumours from 36 patients was studied. Histoscores of FGFR1 immunoreactivity were significantly increased during the $\mathrm{HN}$ to $\mathrm{CR}$ transition for both cytoplasmic (HN vs CR: mean 183 vs 212, median 200 vs 220, respectively, $P=0.005$ ) and nuclear (HN vs CR: mean 194 vs 234, median 200 vs 240, $P=0.002$ ) staining. Membranous FGFR1 staining was not observed in this study, and therefore not included in the analysis. Consistent with previous reports, expression of $\mathrm{AR}$ and Ki67 were significantly increased in CRPC when compared with HNPC $(P<0.0001$; Tan et al, 2010).

To determine whether FGFR1 overexpression is associated with progression of $\mathrm{HN}$ tumours to the $\mathrm{CR}$ state as defined by the time to biochemical relapse (persistent and significant rise in serum PSA levels), Kaplan-Meier graphs for tumours expressing low (below median) and high (above median) levels of FGFR1 were plotted and compared using the log-rank test. Patients with low FGFR1-expressing HNPC had a mean time to relapse of 3.9 years, whereas patients with tumours showing high cytoplasmic FGFR immunoreactivity relapsed quicker with a mean time to relapse of 2.1 years $(P=0.006$; Figure $4 C)$. Nuclear FGFR1 expression was not associated with disease progression.

Kaplan-Meier graphs of tumours were plotted to analyse if enhanced FGFR1 expression in CRPC, when compared with matched HNPC, was related to patient survival outcome. We observed that an increase in (nuclear) FGFR1 expression from HN to CRPC was associated with reduced mean disease-specific survival of 4.9 years, compared with 7.4 years in those that did not show such enhanced FGFR expression in CR tumours $(P=0.018$; Figure $4 \mathrm{D})$. Interestingly, we observed no difference with cytoplasmic expression of FGFR1.

Taken together, upregulated FGFR1 expression is important in prostate carcinogenesis, and we report for the first time its involvement in both HN and CRPC. 
A

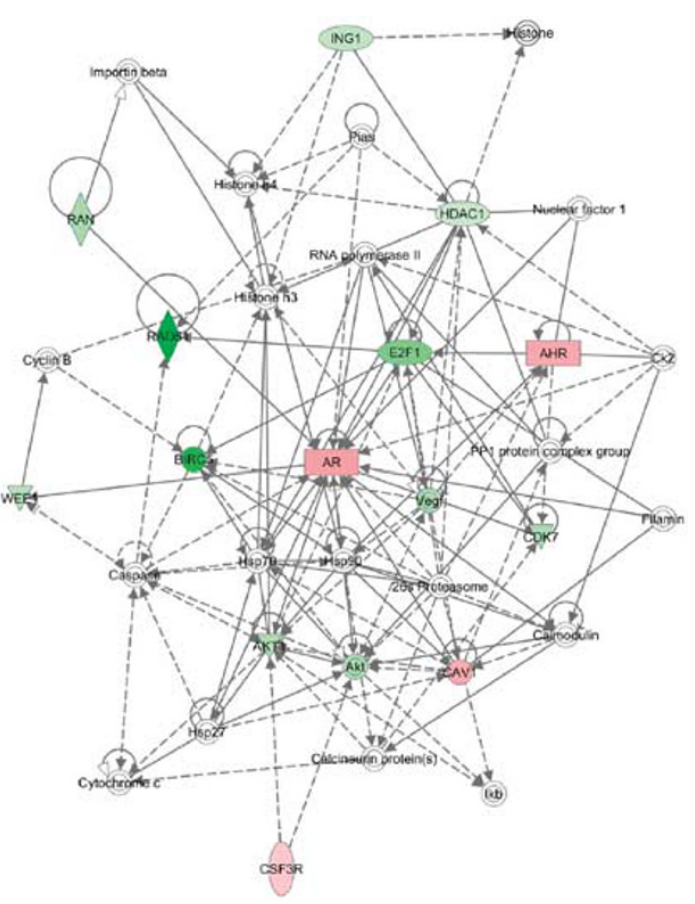

B

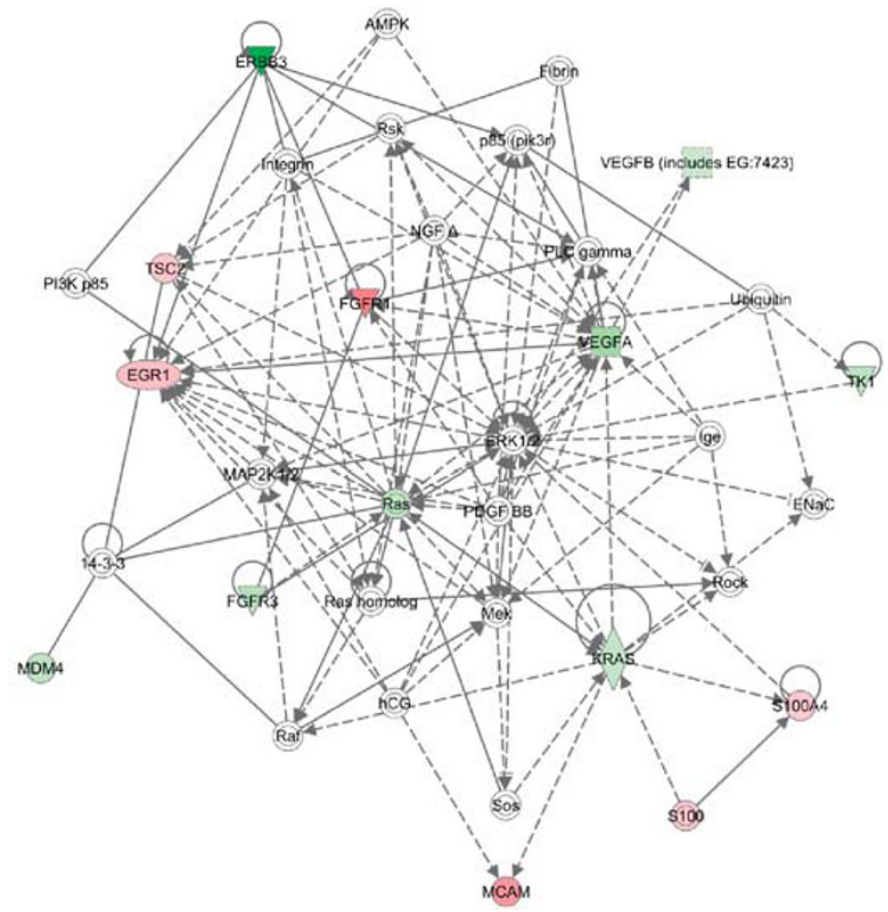

C Network 3

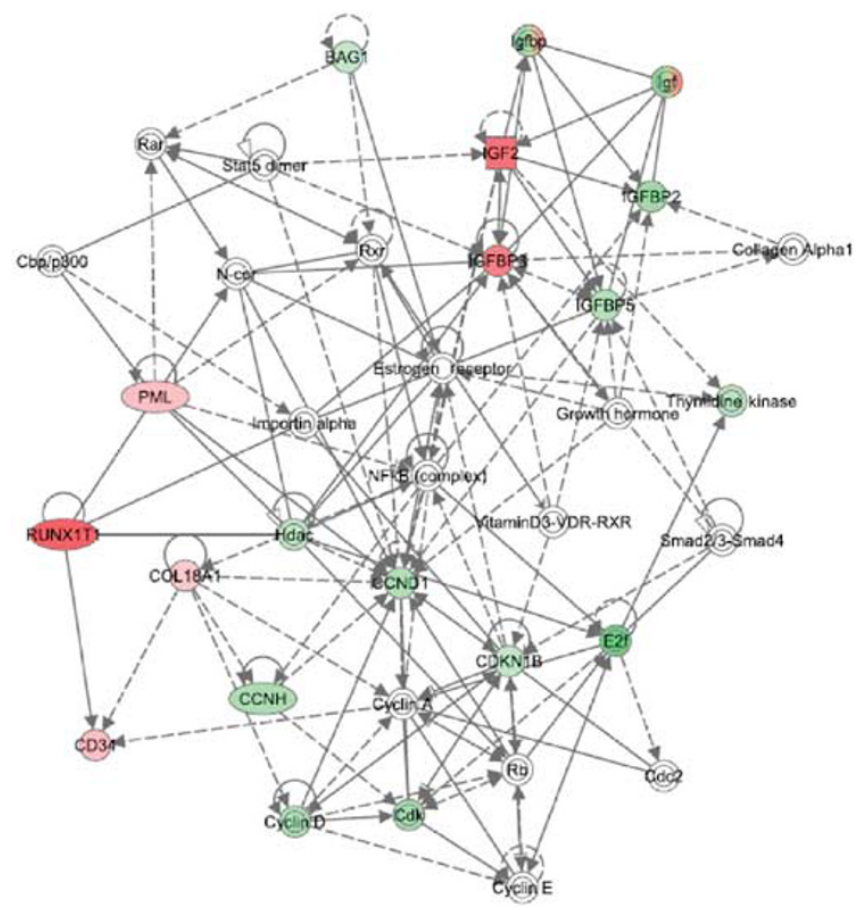

Figure 3 Network I $(\mathbf{A}), 2(\mathbf{B})$ and $3(\mathbf{C})$ were identified by the core analysis using IPA software, to be associated with the HN to CR transition. Details of symbols can be found at http://www.ingenuity.com/. In brief, red and green colours represent input molecules that are upregulated or downregulated, respectively. Additional molecules from the Ingenuity Knowledge Base, shown in white colour, connect the input molecules into a larger meaningful network.

\section{DISCUSSION}

Treatment for CRPC remains unsatisfactory, and novel-targeted therapy is urgently needed. Despite ongoing developments in taxane and androgen ablation therapies, including cabazitaxel and abiraterone, respectively, patients with CRPC continue to die prematurely. We studied matched $\mathrm{HN}$ and CR prostate tumours, using a focused Human Cancer Panel DASL analysis to identify genes involved in the HN to CR transition. Triplicate cores from individual tumour-rich areas were obtained from FFPE samples, and extracted RNA was confirmed to be adequate for analysis, with $\mathrm{Ct}$ values for the house-keeping gene, RPL13a, between 22 and 28 cycles (Bibikova et al, 2004a). Next-generation sequencing methodologies, along with the relevant amplification protocols 
A

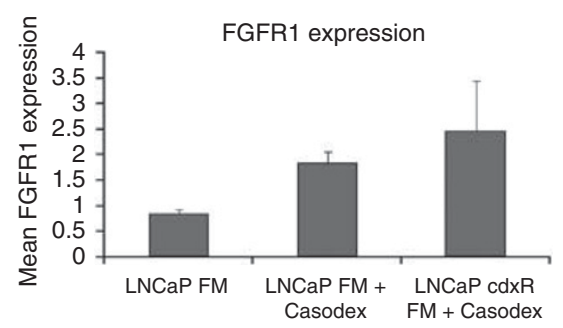

Cell line and growth media

B

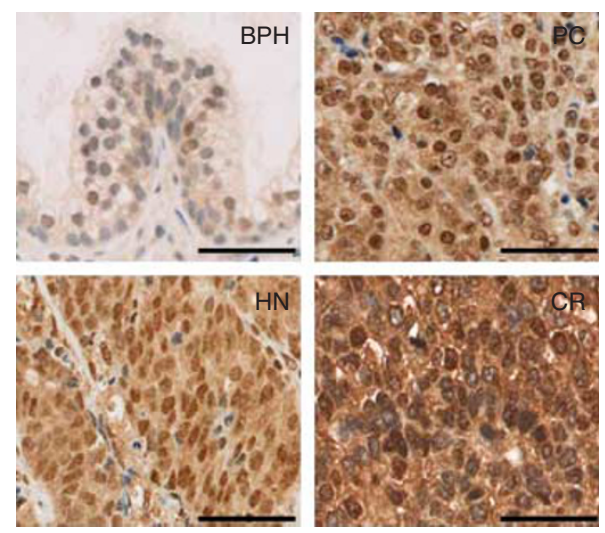

C
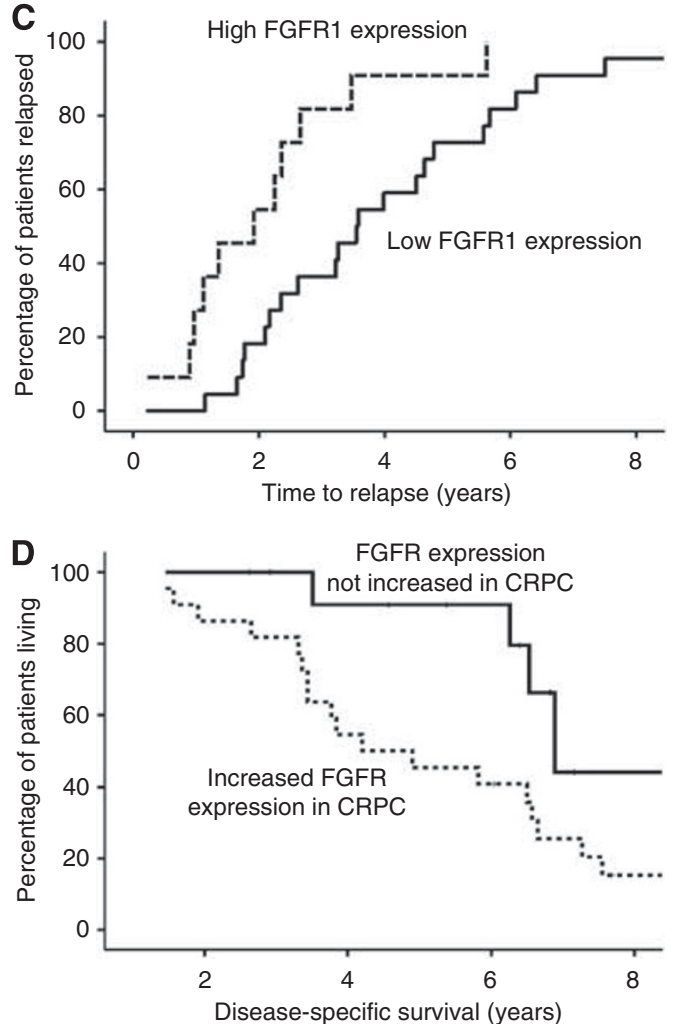

Figure 4 (A) qRT-PCR for FGFRI expression in LNCaP and derived-casodex resistant (cdxR) cells. FGFRI expression was normalised against HPRTI expression and repeated twice. FM denotes full medium growth conditions, and casodex signifies the presence of bicalutamide $(2 \mu \mathrm{M})$. (B) Representative images for FGFRI immunoreactivity in BPH, PC, and paired-HN and -CR tumours. Black bar represent I00 $\mu$ m. (C) Kaplan-Meier graph for the time to biochemical relapse in patients with HNPC as stratified according to FGFRI expression. A total of 33 patients were included in the analysis, 22 classified as having low FGFRI expression (in reference to the median value). By definition, all patients in this cohort had relapsed; therefore, there were also 22 events, as an event was defined by the patients experiencing relapse. In all, I I patients were classified as having high FGFR I expression, and similar to the above reasoning, there were also I I events. (D) Kaplan-Meier graph for disease-specific survival in patients with CRPC as stratified according to whether FGFR I expression was upregulated or not in CR disease, when compared with the respective HN tumours. Follow-up data on patient survival were available for 35 patients and were included in the analysis. In all, 22 patients were classified as having an increase in FGFRI expression with development of CRPC with 20 events and 13 patients were classified as having no change in expression with 5 events. In this case, an event was a cancer-specific death.

are rapidly evolving. Our data highlight the feasibility of studies utilising laser-captured microdissected FFPE materials for genome, exome or RNA sequencing.

A total of 50 out of 502 genes in the cancer panel were significantly different between HN and CR tumours. Overrepresented signalling pathways were identified using the 'core analysis' of the IPA software. The top-ranked network identified AR to be a key component, in keeping with available data on continued AR contribution in CRPC (Edwards et al, 2003; Watson et al, 2010). The second-ranked network implicated FGFR1 to be involved in CRPC. Extending our previous work and reports from other laboratories (Devilard et al, 2006; Sahadevan et al, 2007), we further validated the association of FGFR1 overexpression with CRPC and its impact on clinical outcome: (1) increased risk of developing CRPC with shorter time to relapse when FGFR1 expression is elevated in HN tumours, (2) higher levels in CR tumours when compared with HNPC, and (3) shorter time to death for CRPC with elevated FGFR expression.

The association of nuclear FGFR1 overexpression with reduced patient survival is interesting and warrants further investigation in a larger cohort of patients with CRPC for more robust subgroup survival analysis. It is worth noting that in HNPC, cytoplasmic FGFR1 was associated with disease progression to CRPC, whereas enhanced nuclear FGFR1 expression was found to be related to shorter patient survival in established CR disease. Given the relatively small sample size of this paired TMA, future studies are required to study the significance of nuclear and cytoplasmic FGFR1. Nuclear FGFR1 cooperates to regulate FGF-2, ribosomal S6 kinase (RSK1) and CREB-binding protein, with RSK1 capable of further promoting nuclear localisation of FGFR1 (Dunham-Ems et al, 2006, 2009).

We applied bicalutamide as an in vitro model for androgen ablation therapy. Both acute and chronic bicalutamide-treated LNCaP cells showed significantly upregulated FGFR1 expression ( $>$ two-fold). Interestingly, when cultured in charcoal-striped medium, LNCaP cells completely lost their FGFR1 expression. The underlying mechanism is unclear, but is likely due to the depletion of factors in addition to androgens. Given the close correlation for FGFR1 expression in our in vitro cell model and clinical tumour samples, as well as the specific mode of action for casodex (bicalutmide), we believe that the use of bicalutamide treatment is a valid in vitro tool for modelling androgen ablation.

Among the six networks identified by the core analysis using the IPA software, the top network (with 13 genes) confirmed previous data on the role of AR in CRPC. From network 2, we validated FGFR1 (with the highest fold of upregulation in this network) to be important in CR disease in vitro and in clinical tumours. The relationship between FGFR1 and the other implicated signalling molecules in network 2 warrants further mechanistic analysis, for which the cdxR-LNCaP cells is a useful model. Interestingly, a number of genes identified in network 2 have been implicated in drug resistance in cancers (Mencia et al, 2010; Parra and Ferreira, 
2010). Indeed, FGFR1 itself has been linked with tamoxifen resistance in breast cancer (Turner et al, 2010) and cisplatin resistance in ovarian cancer (Cole et al, 2010). Amplification of FGFR1 has been reported in breast cancers (Turner et al, 2010) and relapsed-PC (Edwards et al, 2003), which may provide a mechanism for the upregulation seen here. Yet, there is still evidence that upregulation of FGFR1 transcription may have a role, based on cell line findings where casodex was applied for $48 \mathrm{~h}$ (Figure 4A). Together, this would result in cells being more sensitive to FGFR1 ligands and potential ligand-independent activation. However, the molecular basis in which FGFR contributes to CRPC requires further analysis to address whether FGFR1 serves a distinct role in $\mathrm{HN}$ and $\mathrm{CR}$ disease. Although network 3 identified 12 genes from the 50 candidate genes, only 3 were upregulated. Nonetheless, it will be important for future studies to explore the impact of these genes (both up- and downregulated) in CRPC.

Prostate-specific activation of FGFR1 in the epithelial compartment results in epithelial-to-mesenchymal transition and development of adenocarcinoma in $100 \%$ of cases. Interestingly, deactivation of FGFR1 in early cancers leads to complete tumour regression, suggesting a role in both initiation and progression (Acevedo et al, 2007), As we have discovered significant association between FGFR1 upregulation and CRPC, future investigation using castration experiments on this transgenic FGFR1 PC mouse model may provide insight into the impact of abnormal FGFR1 function in the development of CRPC. It is also worth noting that despite the earlier observation of in vitro activation of the AR by growth factors, including members of the FGF family (Culig et al, 1994), whether aberrant FGFR signalling may contribute to prostate carcinogenesis via ligand-independent activation of AR remains unclear. To our knowledge, somatic mutations of FGFR1 are not involved in human PC. Interestingly, miR-16 is thought to regulate several target genes including FGFR1 (Gatt et al, 2010) and may be responsible for aberrant FGFR1 expression in PC. Future investigations can assess if miR-16 expression is progressively lost with the transition from $\mathrm{HN}$ to $\mathrm{CR}$ disease. The three-fold induction of FGFR 1 expression observed in the development of CRPC is the highest among the genes activated in the second-ranked network, and along with the reported contribution of FGFR1 in PC biology, we would propose that FGFR1 is the gene of interest rather than a proxy or downstream effect of an independent tumour-promoting event.

In summary, our gene expression analysis using a focused cancer-associated gene set supported previous data on $A R$ and uncovered the association of FGFR1 in CRPC.

\section{ACKNOWLEDGEMENTS}

This project was funded by NCRI PROMPT (Grant number: G0100100/64424), Cancer Research UK, and the Beatson Institute for Cancer Research. IA is a recipient of MRC and CRUK Clinical Training Fellowship. We thank Jacqueline Stockley, Steven Darby and $\mathrm{K}$ Sahadevan for technical assistance.

\section{Author contribution}

This study was designed by HYL, and data was collected by KA, ST and IA. Data analysis was performed by JE, IA, GK and HYL. Data was interpreted by CNR and HYL. Literature searches were performed by KA, IA and HYL. Figures were generated by KA, IA and HYL. This manuscript was written by KA and HYL.

\section{Additional information}

Raw data from gene expression analysis has been uploaded online as supplementary supporting information. Briefly, data for individual patient samples are shown for each gene present on the bead array, including signal from the array, detection score (to indicate whether the target sequence signal is distinguishable from the negative control), fold change between naive and relapse samples and DiffScore to describe the significance of the fold change observed.

Supplementary Information accompanies the paper on British Journal of Cancer website (http://www.nature.com/bjc)

\section{REFERENCES}

Acevedo VD, Gangula RD, Freeman KW, Li R, Zhang Y, Wang F, Ayala GE, Peterson LE, Ittmann M, Spencer DM (2007) Inducible FGFR-1 activation leads to irreversible prostate adenocarcinoma and an epithelial-tomesenchymal transition. Cancer Cell 12(6): 559-571

Aggarwal R, Ryan CJ (2011) Castration-resistant prostate cancer: targeted therapies and individualized treatment. Oncologist 16(3): 264-275

Berger W, Setinek U, Mohr T, Kindas-Mugge I, Vetterlein M, Dekan G, Eckersberger F, Caldas C, Micksche M (1999) Evidence for a role of FGF-2 and FGF receptors in the proliferation of non-small cell lung cancer cells. Int J Cancer 83(3): 415-423

Bibikova M, Talantov D, Chudin E, Yeakley JM, Chen J, Doucet D, Wickham E, Atkins D, Barker D, Chee M, Wang Y, Fan JB (2004a) Quantitative gene expression profiling in formalin-fixed, paraffinembedded tissues using universal bead arrays. Am J Pathol 165(5): $1799-1807$

Bibikova M, Yeakley JM, Chudin E, Chen J, Wickham E, Wang-Rodriguez J, Fan JB (2004b) Gene expression profiles in formalin-fixed, paraffinembedded tissues obtained with a novel assay for microarray analysis. Clin Chem 50(12): 2384-2386

Bibikova M, Yeakley JM, Wang-Rodriguez J, Fan JB (2008) Quantitative expression profiling of RNA from formalin-fixed, paraffin-embedded tissues using randomly assembled bead arrays. Methods Mol Biol (Clifton, NJ) 439: 159-177

Chudin E, Kruglyak S, Baker SC, Oeser S, Barker D, McDaniel TK (2006) A model of technical variation of microarray signals. J Comput Biol 13(4): 996- 1003

Cole C, Lau S, Backen A, Clamp A, Rushton G, Dive C, Hodgkinson C, McVey R, Kitchener H, Jayson GC (2010) Inhibition of FGFR2 and FGFR1 increases cisplatin sensitivity in ovarian cancer. Cancer Biol Ther 10(5): $495-504$

Culig Z, Hobisch A, Cronauer MV, Radmayr C, Trapman J, Hittmair A, Bartsch G, Klocker H (1994) Androgen receptor activation in prostatic tumor cell lines by insulin-like growth factor-I, keratinocyte growth factor, and epidermal growth factor. Cancer Res 54(20): $5474-5478$

Devilard E, Bladou F, Ramuz O, Karsenty G, Dales JP, Gravis G, Nguyen C, Bertucci F, Xerri L, Birnbaum D (2006) FGFR1 and WT1 are markers of human prostate cancer progression. BMC Cancer 6: 272

Dunham-Ems SM, Lee YW, Stachowiak EK, Pudavar H, Claus P, Prasad PN, Stachowiak MK (2009) Fibroblast growth factor receptor-1 (FGFR1) nuclear dynamics reveal a novel mechanism in transcription control. Mol Biol Cell 20(9): 2401-2412

Dunham-Ems SM, Pudavar HE, Myers JM, Maher PA, Prasad PN, Stachowiak MK (2006) Factors controlling fibroblast growth factor receptor-1's cytoplasmic trafficking and its regulation as revealed by FRAP analysis. Mol Biol Cell 17(5): 2223-2235

Edwards J, Krishna NS, Witton CJ, Bartlett JM (2003) Gene amplifications associated with the development of hormone-resistant prostate cancer. Clin Cancer Res 9(14): $5271-5281$

Fan JB, Yeakley JM, Bibikova M, Chudin E, Wickham E, Chen J, Doucet D, Rigault P, Zhang B, Shen R, McBride C, Li HR, Fu XD, Oliphant A, Barker DL, Chee MS (2004) A versatile assay for high-throughput gene 
expression profiling on universal array matrices. Genome Res 14(5): $878-885$

Ferlay J, Autier P, Boniol M, Heanue M, Colombet M, Boyle P (2007) Estimates of the cancer incidence and mortality in Europe in 2006. Ann Oncol 18(3): $581-592$

Gatt ME, Zhao JJ, Ebert MS, Zhang Y, Chu Z, Mani M, Gazit R, Carrasco DE, Dutta-Simmons J, Adamia S, Minvielle S, Tai YT, Munshi NC, Avet-Loiseau $\mathrm{H}$, Anderson KC, Carrasco DR (2010) MicroRNAs 15a/16-1 function as tumor suppressor genes in multiple myeloma. Blood 117(26): 7188

Halkidou K, Gnanapragasam VJ, Mehta PB, Logan IR, Brady ME, Cook S, Leung HY, Neal DE, Robson CN (2003) Expression of Tip60, an androgen receptor coactivator, and its role in prostate cancer development. Oncogene 22(16): 2466-2477

Mencia N, Selga E, Rico I, de Almagro MC, Villalobos X, Ramirez S, Adan J, Hernandez JL, Noe V, Ciudad CJ (2010) Overexpression of S100A4 in human cancer cell lines resistant to methotrexate. BMC Cancer 10: 250

Parra E, Ferreira J (2010) The effect of siRNA-Egr-1 and camptothecin on growth and chemosensitivity of breast cancer cell lines. Oncol Rep 23(4): $1159-1165$

Reid AH, Attard G, Danila DC, Oommen NB, Olmos D, Fong PC, Molife LR, Hunt J, Messiou C, Parker C, Dearnaley D, Swennenhuis JF, Terstappen LW, Lee G, Kheoh T, Molina A, Ryan CJ, Small E, Scher HI, de Bono JS (2010) Significant and sustained antitumor activity in post-docetaxel, castration-resistant prostate cancer with the CYP17 inhibitor abiraterone acetate. J Clin Oncol 28(9): 1489-1495
Rigas AC, Robson CN, Curtin NJ (2007) Therapeutic potential of CDK inhibitor NU2058 in androgen-independent prostate cancer. Oncogene 26(55): $7611-7619$

Sahadevan K, Darby S, Leung HY, Mathers ME, Robson CN, Gnanapragasam VJ (2007) Selective over-expression of fibroblast growth factor receptors 1 and 4 in clinical prostate cancer. J Pathol 213(1): $82-90$

Scher HI, Morris MJ, Kelly WK, Schwartz LH, Heller G (2005) Prostate cancer clinical trial end points: 'RECIST' ing a step backwards. Clin Cancer Res 11(14): 5223-5232

Shen HC, Balk SP (2009) Development of androgen receptor antagonists with promising activity in castration-resistant prostate cancer. Cancer Cell 15(6): $461-463$

Tan SS, Ahmad I, Bennett HL, Singh L, Nixon C, Seywright M, Barnetson RJ, Edwards J, Leung HY (2010) GRP78 up-regulation is associated with androgen receptor status, Hsp70-Hsp90 client proteins and castrateresistant prostate cancer. $J$ Pathol 223(1): $81-87$

Turner N, Pearson A, Sharpe R, Lambros M, Geyer F, Lopez-Garcia MA, Natrajan R, Marchio C, Iorns E, Mackay A, Gillett C, Grigoriadis A, Tutt A, Reis-Filho JS, Ashworth A (2010) FGFR1 amplification drives endocrine therapy resistance and is a therapeutic target in breast cancer. Cancer Res 70(5): 2085-2094

Watson PA, Chen YF, Balbas MD, Wongvipat J, Socci ND, Viale A, Kim K, Sawyers CL (2010) Constitutively active androgen receptor splice variants expressed in castration-resistant prostate cancer require fulllength androgen receptor. Proc Natl Acad Sci USA 107(39): 16759-16765

This work is published under the standard license to publish agreement. After 12 months the work will become freely available and the license terms will switch to a Creative Commons Attribution-NonCommercial-Share Alike 3.0 Unported License. 